\title{
BMJ Open Protocol for a non-randomised feasibility study evaluating a codesigned patient safety guide in primary care
}

\author{
Rebecca L Morris (D) , ${ }^{1}$ Kay Gallacher, ${ }^{2}$ Mark Hann, ${ }^{3}$ Carly Rolfe, ${ }^{1}$ Nicola Small (i) , ${ }^{4}$ \\ Sally J Giles, ${ }^{1}$ Caroline Sanders, ${ }^{1}$ Stephen M Campbell ${ }^{1}$
}

To cite: Morris RL, Gallacher K, Hann M, et al. Protocol for a non-randomised feasibility study evaluating a codesigned patient safety guide in primary care. BMJ Open 2021;11:e039752. doi:10.1136/ bmjopen-2020-039752

- Prepublication history and additional materials for this paper is available online. To view these files, please visit the journal online (http://dx.doi. org/10.1136/bmjopen-2020039752).

Received 24 April 2020

Revised 17 December 2020

Accepted 26 December 2020

Check for updates

(c) Author(s) (or their employer(s)) 2021. Re-use permitted under CC BY-NC. No commercial re-use. See rights and permissions. Published by BMJ.

${ }^{1}$ NIHR Greater Manchester Patient Safety Translational Research Centre, The University of Manchester, Manchester, UK

${ }^{2}$ Manchester, UK

${ }^{3}$ Centre for Biostatistics and Manchester Academic Health Science Centre, The University of Manchester, Manchester, UK

${ }^{4}$ The University of Manchester, Manchester, UK

\section{Correspondence to}

Dr Rebecca L Morris;

rebecca.morris@manchester. ac.uk

\section{ABSTRACT}

Introduction Patients and carers should be active partners in patient safety with healthcare professionals and be empowered to use personalised approaches to identify safety concerns and work together to prevent them. This protocol paper details a study to examine the feasibility of a multicomponent intervention to involve patients and/or carers in patient safety in primary care in the UK.

Methods and analysis This is a two-phase, nonrandomised feasibility mixed methods pragmatic study of a patient safety guide for primary care (PSG-PC). 8 general practices will recruit 120 patient and/or carer participants. All patient and/or carer participants will receive the PSGPC. It will examine the feasibility and acceptability of the PSG-PC in primary care settings in patients aged 18 years or older who attend appointments at general practice with health professionals four or more times per year as either patients or carers. It will identify secondary outcomes for improving patient safety, health status and patient empowerment, and reducing health service utilisation over 6 months between baseline and 6-month follow-ups. The findings will inform whether a main effectiveness trial is feasible and, if so, how it should be designed, and how many patients and practices will be needed. The study will be undertaken between January 2020 and September 2021.

Ethics and dissemination Ethical approval was obtained from the National Health Service London-West London and Gene Therapy Advisory Committee Research Ethics Committee (reference: 19/L0/1289). Research findings will be disseminated with participating general practices and shared in a range of different ways to engage different audiences, including presenting at international and national conferences, publishing in open-access, peer-reviewed journals and facilitating dissemination workshops within local communities with patients, carers and healthcare professionals.

Trial registration number ISRCTN90222092.

\section{INTRODUCTION}

Patient safety is a global priority and it requires commitment and action at all levels of the health and social care system from governments down to individual patients and healthcare professionals. ${ }^{12}$ There has been a move from a medicolegal, culpability-focused

\section{Strengths and limitations of this study}

- First study evaluating the patient safety guide for primary care delivered via a paper and mobile app in primary care.

- This study will examine the role of involving patients actively in patient safety which has remained underexplored in primary care.

- Qualitative and quantitative approaches will be used to comprehensively assess study outcomes to inform a main trial and whether it would be feasible.

- A potential limitation of this study is a lack of randomisation of the sample, rather it will assess descriptively whether it has any impact by comparing data before and after receiving the guide.

- The outcomes to be assessed by this study are relevant to patients, carers, primary care clinicians and policymakers.

approach towards a recognition of the need to understand and learn from incidents to avoid them from happening again. ${ }^{34}$ While much of the research focus for patient safety has been on secondary care, the volume of patient contact with primary care makes understanding implementation of patient safety initiatives in this setting a priority. ${ }^{56}$ In the UK, the National Patient Safety Strategy stressed the role of government, organisations like National Health Service (NHS) England and by the 'untapped potential' of involving patients and carers in patient safety. ${ }^{2} 78$ The aim is to enhance safety beyond the prevention of serious incidents by preventing harm before it occurs and see risks and make them safe. ${ }^{2}$

The role of patients and carers in safety can be seen within three main areas ${ }^{9}$ :

1. Patients monitoring the progress and effects of their treatment.

2. Patients informing clinicians about how to modify their personal care plans.

3. Patients informing services about how to improve clinical practices. ${ }^{6}$ 
Involving different stakeholders in patient safety has to recognise that perceptions of safety and harm may differ with patients placing a greater emphasis on the wider psychosocial components of care and not just serious incidents. ${ }^{3} 10-12$ One approach to incorporate patients' views about patient safety is to build trust, clarify expectations and ensure understanding. ${ }^{12}{ }^{13}$ The focus of the majority of research on patient safety in primary care has been descriptive, with few studies focusing on interventions that will improve it or the role of patients within this. ${ }^{14}{ }^{15}$ Potential patient safety issues may be identified along patient pathways from access, to diagnosis, to treatment and self-management with primary care managing repeated uncertainties along episodes of care. ${ }^{316-19}$

An individual patient or carer's understanding of both patient safety and the healthcare system is interdependent. This is shaped by previous experiences with healthcare services and has implications for clinical interactions shaping an individual's capacity or willingness to raise safety concerns especially where previous experiences have left patients feeling vulnerable and powerless. ${ }^{70-23}$ For example, increasingly research suggests that patients living with multimorbidity or more complex care needs have many difficulties coping with daily activities and poorer quality of life, and experience duplication and fragmentation of care. ${ }^{24-26}$ As such, involving patients in patient safety requires a collaborative approach to address potential barriers as well as to recognise that there may be multiple interacting influences affecting safety issues or solutions to address them. ${ }^{27}$ However, this is a shift expectation of the role of patients in patient safety needs to be evaluated in order to investigate unintended consequences that could compound patient safety risks. ${ }^{28}$

The patient safety guide for primary care (PSG-PC) supports patients and carers to address key patient safety questions and identify key points where they can make their care safer and answer key patient safety questions. It has been developed using extensive codesign in partnership with patients, carers, members of the public, and healthcare professionals including general practitioners (GPs) and pharmacists, and has been reported in detail elsewhere. ${ }^{29} 30$ The PSG-PC was codeveloped to support effective communication between patients and clinicians, which was considered a key component of involving patients and carers in patient safety. ${ }^{10}$

In accordance with the Medical Research Council framework for the design and evaluation of complex interventions, this study is the next stage in the programme of work. ${ }^{31}$ The aim of this study is to test the PSG-PC (which is available on paper, a web-based platform and as an app) with patients, carers and healthcare professionals within primary care. A quantitative and qualitative feasibility evaluation will be undertaken to gain an in-depth understanding of a multifactorial intervention. ${ }^{32}$

The primary objectives of the feasibility study are:

1. To determine the feasibility and acceptability of the PSG-PC in primary care settings in patients aged 18 years or older who attend appointments at general practice with health professionals four or more times per year as either patients or carers.

2. To determine how many people accept the invitation to participate in the study.

3. To estimate recruitment and refusal rates and 6-month attrition rates.

4. To investigate which formats of the PSG-PC (app, paper or website) are acceptable (if any), to whom (patients, subgroups of patients, carers, health professionals (subgroups) ) and in what circumstances.

5. To examine the factors which influence the use of the PSG-PC for patients and carers within primary care.

The secondary objectives are:

1. To descriptively investigate whether the PSG-PG improves patient safety, health status and patient empowerment, and reduces health service utilisation over 6 months.

2. To investigate whether the PSG-PC influences patients and carers' views of patient safety and patient empowerment.

3. To assess study outcomes to inform a main trial and whether it would be feasible.

\section{METHODS AND ANALYSIS}

\section{Study design}

This feasibility study is a non-randomised study and all participants will receive the intervention. An explanatory sequential mixed methods approach will be used to assess feasibility. ${ }^{33}$ The feasibility study will be in line with the guidance proposed by Eldridge $e t a l^{34}$ and reported using the Standard Protocol Items: Recommendations for Interventional Trials reporting template (see online supplemental files 1-3). ${ }^{35}$

\section{Intervention}

The study will comprise two phases. Phase 1 will include two initial 'pilot' general practices to initially pilot the PSG-PC and identify initial acceptability and inform feasibility questions for phase 2 (such as the number of patients needed to be contacted to reach the required participant numbers in phase 2).

There are two key components of the intervention:

A. Healthcare professional and practice staff introductory workshop: An introductory workshop, facilitated by the research team and members of the study public involvement group, will be held with general practice staff where the purpose of the PSG-PC will be outlined. The workshop, lasting approximately 1 hour, will be held in each general practice in both phase 1 and phase 2 with clinical (GP, practice nurse) and non-clinical staff (practice manager and receptionist).

B. The PSG-PC includes: (1) the PSG-PC including the short paper version, (2) a website (www.patientsafetyguide.org), and (3) a digital app (developed to work on both iOS and Android platforms). 


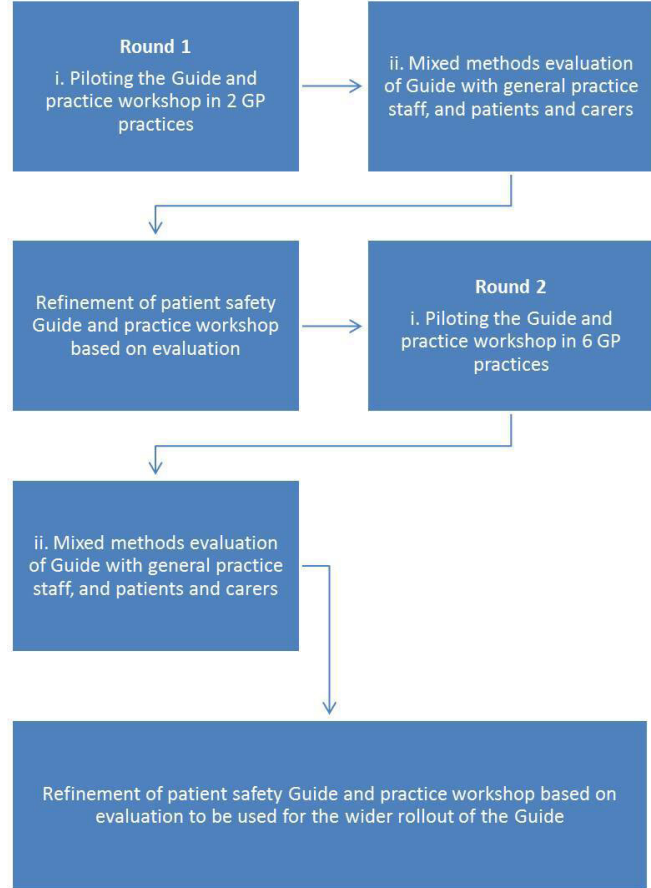

Figure 1 Process flow of study design and intervention. GP, general practitioner.

\section{Participants}

The study will be conducted in two phases (see figure 1). As the components of the intervention are novel, we proposed a two-phase internal approach to ensure: (A) that there are no serious adverse events as a result of using the PSG-PC; (B) feasibility of recruiting practices to the study; and $(\mathrm{C})$ feasibility of recruiting patients. The aim of a two-phase approach is to do an initial proof of concept and to stop the study from progressing to phase 2 if it does not meet those criteria. ${ }^{36}$

As this is a feasibility study, a formal sample size calculation is not required. ${ }^{37}$ We will aim to recruit 120 patients across both phases (to complete questionnaires and 30 patients sampled within this for interviews). Sixteen healthcare professionals across phase 1 and phase 2 will be recruited for interviews. In phase 1, two general practices will be recruited to initially pilot the intervention, baseline, postal questionnaire and 6-month follow-up data collection (the outcomes measured by the questionnaires are detailed in the section Outcomes to be Measured at Baseline and 6-month Follow-up). In phase 2 , six general practices will be recruited. We will aim to recruit 15 patients at each practice to address feasibility issues (recruitment, retention, attrition, and so on), estimate parameters needed to conduct a power calculation for a full trial and to be able to explore the performance of the outcome measures. Quantitative methods will focus on the completion rates of validated self-report questionnaires (at baseline and 6-month follow-up questionnaires) to look at acceptability of questionnaires to inform the choice of outcome measures in a larger randomised controlled trial as well as how often, and in what ways, participants report using the PSG-PC. Participants will be given a unique identification number and data will be treated with confidentiality and stored securely at the Centre for Primary Care and Health Services Research, the University of Manchester.

\section{Eligibility criteria}

Participants are eligible for this study (patients and/or carers and health professionals) if they are aged 18 or over, must attend appointments with health professionals four or more times per year and be able to read and converse in English. Additionally, patient participants will be sampled with a variety and number of long-term conditions (including diabetes).

Patients who are currently residing in hospital or in nursing homes will be excluded since this is not the focus of this study. Patients who are unable to read or write English will be unable to use the PSG-PC (in its current format). Patients on an end-of-life care pathway will also be ineligible to minimise additional burden on them. Screening for eligibility will take place by GPs and appropriate practice staff.

\section{Recruitment and data collection}

Phase 1

\section{General practice sites}

Practices will be recruited by the research team through invitation letters to research active practices. Two initial 'pilot' general practices will be recruited for phase 1. An initial letter of invitation outlining the study will be sent to research active practices within Greater Manchester. The study researcher will follow-up interest from practices to facilitate engagement and further understanding of the study. Practices will be reimbursed for $£ 500$ to cover clinician and administration time for participating in this study and for identifying potential patient participants. To be included in the study, practices must be based in Greater Manchester.

\section{Patient and/or carer participants}

Fifteen patients and/or carers per practice will be recruited by staff based on the practice and invited by letter to take part in the research study. In order to ensure patients and/or carers are included who are regularly visiting their GP practice, staff will select patients and/or carers who have seen healthcare professionals at the practice at least four times over the past year for a range of types and numbers of long-term conditions (such as diabetes, chronic obstructive pulmonary disease or multiple sclerosis). Practice staff will identify suitable patients and/or carers from their computerised practice lists who meet the inclusion criteria.

Patients will be invited to participate by the baseline questionnaire with a brief patient information sheet included along with the consent to contact form (the outcomes measured by the questionnaires are detailed in the section Outcomes to be Measured at Baseline and 6-month Follow-up). The questionnaires include questions on patient safety, patient empowerment and 
health service utilisation. Completion and returning the questionnaire will be classed as implied consent to test the PSG-PC intervention. Phase 1 will inform the approximate number of patients who are contacted by the practice to generate 15 consented participants. Once the completed baseline questionnaire and consent to contact form has been received, the participant will be sent a paper copy of the PSG-PC and information about how to download digital versions of the PSG-PC as well as their $£ 5$ gift voucher for completing the questionnaire. Six months later, a follow-up questionnaire will be sent to participants with an optional reminder to be sent 2 weeks later if the questionnaire has not been returned. Included in the final questionnaire will be an opt-in form for participants to volunteer to be interviewed. After the final questionnaire has been completed and returned, a letter of thanks and a $£ 5$ voucher will be sent to each participant. As participation in the research is voluntary, participants can withdraw consent at any time without giving any reason without their care or legal rights being affected. If a participant who has given informed consent loses capacity to consent during the study, the participant would be withdrawn from the study. However, data already collected with consent would be retained and used in the study.

\section{Qualitative patient participant interviews}

After 6 months, a sample of approximately 10 patients and/or their carers from phase 1 will be invited to take part in interviews about using the guide. Participants will express their interest to participate by completing the expression of interest form at the end of the 6-month questionnaire. Participants will be purposively sampled to take part in interviews based on the demographics (eg, age, gender and ethnicity) and a variety of number and types of long-term conditions. ${ }^{38}$ Interviews will explore how the PSG-PC has been used, by whom and which versions have been employed and will gather any other feedback useful for future roll-out. Participants will have been given the information sheet at least 24 hours before the interviews and will provide written consent to take part before the interview begins. Any questions about participation will be answered by the research team. Interviews will last approximately $40 \mathrm{~min}$ and will take place at a time and location that is convenient for the participant. Patient and/or carer participants will receive a £20 shopping voucher for participating in the interview. Verbatim transcripts of the audio-recorded interviews will be analysed over the course of data collection.

\section{Healthcare professional and practice staff level}

Practice staff will be invited to attend introductory workshops and representatives from all staff groups (clinical including GPs and nurse, and non-clinical including receptionists and practice managers) and a purposive sample invited to take part in qualitative interviews about the patient safety guide. After the initial introductory workshop, practice staff will be asked to complete a brief evaluation of the workshop. At the end of the phase 1 6-month period, practice staff (including healthcare professionals) at participating practices will be asked to express interest in taking part in an interview and will be given the healthcare professional and practice staff information sheet before agreeing to take part in an interview. A purposeful sample of around six practice staff covering different staff groups will be interviewed to seek feedback about the workshop and the acceptability of the patient safety guide, its functionality, format and design, as well as to understand the facilitators and barriers to using the PSG-PC in everyday practice. ${ }^{37}$ Participants will be given the information sheet at least 24 hours before the interviews and will provide written consent to take part before the interview begins. Any questions about participation will be answered by the research team at any time. Participants will be reimbursed with vouchers for participating in the interviews. Participants will be reimbursed according to staff type: a GP will receive $£ 100$, a nurse $£ 50$, a pharmacist $£ 50$ and other general practice staff $£ 30$. Interviews will take place at a time that is convenient for the participant.

\section{Phase 2}

A further six general practices will be recruited in the same way. The introductory workshops will be amended following phase 1 feedback. All other work will continue in the same way as in phase 1 .

At the end of phase 2 , similarly around 10 practice staff and 20 patients and/or carers will be interviewed with the same approach adopted in phase 1 for recruitment and data collection.

\section{Outcomes to be measured at baseline and 6-month follow-up}

The aim of the evaluation will be to determine the feasibility and acceptability of the PSG-PC used by patients, in what ways and to identify if there are any changes in relation to key outcomes (see figure 2). As this is a nonrandomised feasibility study it will not be able to measure effectiveness but rather will look at whether the PSG-PC is acceptable and, if so, for whom.

The quantitative outcomes will be measured using two paper postal surveys: one at baseline and a second at 6-month follow-up. The primary measure that will be used to examine patient-reported experience of patient safety will be the shortened version of the Primary Care Patient Measure of Safety. ${ }^{39}$ It is a 25-item measure developed for patients to provide feedback about factors contributing to potential safety incidents. ${ }^{39}$ It was developed as a way for primary care organisations to use feedback from patients to learn about the contributory factors to patient safety and make service improvements. Patient empowerment will be measured at baseline and 6-month follow-up using the 47-item measure developed and preliminarily validated to measure patient empowerment in predominately older patients with multiple long-term health conditions in primary care. ${ }^{24}$ Data on service use and health status will be collected at baseline and follow-up 


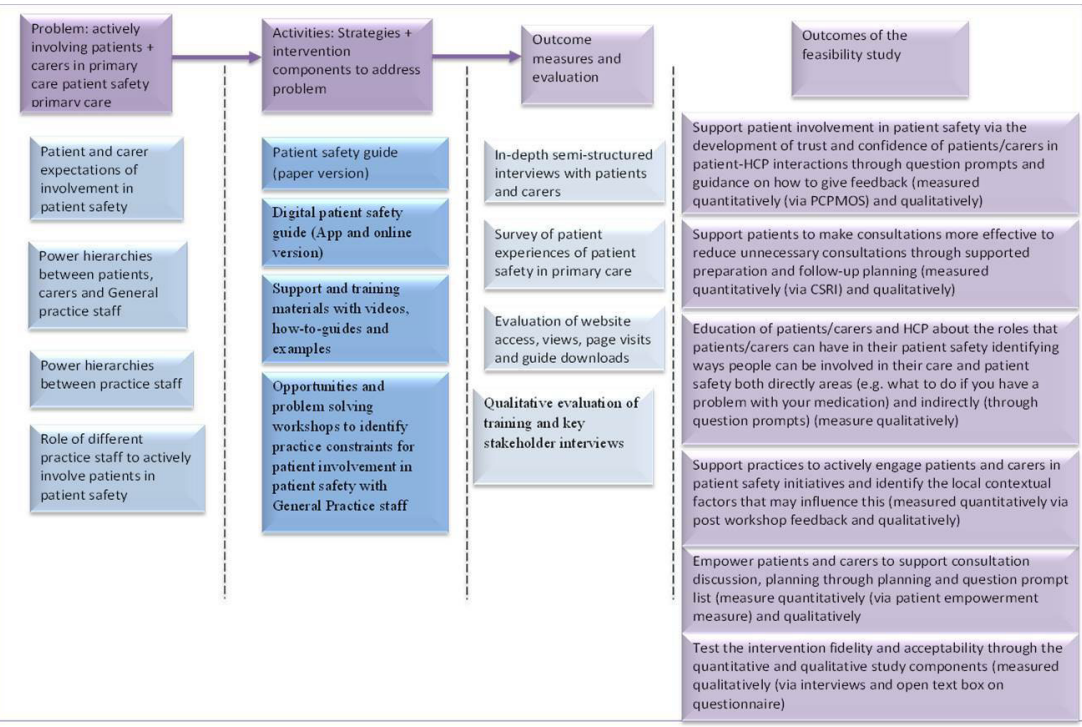

Figure 2 Logic model for study and intervention. CSRI, Client Service Receipt Inventory; HCP, healthcare professional; PCPMOS, Primary Care Patient Measure of Safety.

using the Client Service Receipt Inventory and 5-Level version of EuroQol-5 Dimension (EQ-5D-5L), respectively. ${ }^{41}$ This will be used at baseline and follow-up at 6-month time point with the same patients. Demographic data (collected at baseline) and primary and secondary measures (collected at baseline and 6-month follow-up) will be collected via a postal survey. The follow-up questionnaire will also include questions evaluating participants' use of the guide, which formats they used and a free text box for feedback. The outcomes to be measured were identified with the public involvement group and GP and pharmacy involvement contributors.

Analysis will use descriptive statistics to examine if people have used the PSG-PC and in what ways by examining changes in responses between baseline and follow-up as well as completion rates of individual questions. At follow-up, feedback will also be asked from participants about what they thought of the guide, whether they used it, what formats and any changes they would suggest to inform the development of the guide.

\section{Healthcare professional and practice staff introductory workshop evaluation}

Feedback will be collected after the workshops and used to inform phase 2 workshops with the introductory workshop evaluation form.

\section{In-depth qualitative evaluation}

A qualitative process evaluation will be conducted using semistructured interviews in order to provide understanding of study delivery and scale-up of the intervention roll-out. A purposeful sample from within the participants of patients and/or carers $(n=30)$ and practice staff $(n=16)$ will be interviewed at 6 months to examine the usability, acceptability and practicality of using the PSG-PC by patients and healthcare professionals over time. ${ }^{38}$ Sampling for participants will be informed by the quantitative data to sample participants based on a range of characteristics including age, gender, ethnicity, longterm conditions (for patients) and/or professional roles (for healthcare professionals) and use of the PSG-PC. The interviews with all stakeholders will be used to inform the refinement of the PSG-PC and workshop.

\section{Data analysis plan}

Statistical analysis

We will report, with 95\% CIs where appropriate, recruitment rates, attrition rates and questionnaire completion rates, along with any observed floor and ceiling effects. Descriptive statistics (eg, as appropriate, mean/median; $\mathrm{SD} / \mathrm{IQR}$; frequency, proportion and $95 \% \mathrm{CI}$; range) will be used to explore the quantitative data (baseline, follow-up and changes from baseline) as this is a feasibility study and so not powered for inferential tests of significance. If possible, we will also estimate the intrapractice correlation coefficient for potential primary outcome measures, as this will be required in a future power calculation. Health economic costs will be estimated as quantity of service used multiplied by the unit cost of that service, for each item of the service used (in this case primary care only). The total cost per patient will be estimated. The health status data from the EQ-5D-5L will be used to estimate the utility index score using published tariffs for the EQ-5D. The service use, cost and utility data will be analysed descriptively to estimate the mean, SD and $95 \%$ CI. The feasibility of the study will also be assessed if it has been able to recruit within an appropriate amount of time (15 patients per practice in 2 months).

\section{Qualitative analysis}

Verbatim anonymised transcripts of the audio-recorded interviews will be analysed over the course of data collection. Analysis will be thematic and relevant categories and concepts will be identified. ${ }^{42}{ }^{43}$ Atlas.ti will be used to 
aid data organisation and analysis. This approach will be theoretically informed by normalisation process theory to understand how information and resources are shared, innovation is shared and the work of implementation. ${ }^{44} 45$

\section{Integration of quantitative and qualitative data}

The quantitative and qualitative results will be connected; we will consider the quantitative and qualitative analyses separately and how they converge or differ in their findings when presenting the overall conclusion. ${ }^{46}$ The qualitative data collected across the two phases will be used to help explain or elaborate on the quantitative data. ${ }^{46}$ This will occur at the end of phase 1 and phase 2 and then will be combined at the end of the study to explain the overall findings. ${ }^{47}$

\section{Patient and public involvement}

Patient and public involvement has occurred throughout the project building on the approach adopted for the initial PSG-PC development. ${ }^{29} 30$ There is a core patient and public contributor group with six members and a patient and public involvement lead (KG). The involvement group worked closely with RLM to develop the materials and will continue to do so throughout the project to support recruitment, data collection, analysis, interpretation and dissemination. Two members of the public contributor group will also be involved in the introductory workshop at general practices and explaining why they feel the PSG-PC is important for patients and carers and helping to identify solutions to any implementation issues. Contributors will be reimbursed in line with the NIHR Greater Manchester Patient Safety Translational Research Centre and NIHR INVOLVE (a national advisory group supporting active patient and public invovlement in NHS, public health and social care research) guidelines. ${ }^{48}$ Any training or support needs identified will be provided.

\section{Ethics}

Ethical approval was obtained from the NHS LondonWest London and Gene Therapy Advisory Committee Research Ethics Committee (reference: 19/LO/1289). Patient participants will provide implied consent by returning the baseline questionnaire and contact details to the study team. For the qualitative interviews, participants will be asked if they would like to take part and provided the relevant participant information sheet at least 24 hours before an interview. Written consent will be given before an interview.

\section{RESULTS}

The study began in January 2020 and is expected to end in June 2021. Due to the onset of the global pandemic of COVID-19 since the start of recruitment for this study, all NHS research recruitment activity has stopped, research will continue in line with government, local and funder guidance. $^{49}$

\section{Dissemination}

The results will be shared with participating general practices and shared with a range of different ways to engage different audiences including presentations at international and national conferences, and publishing in open-access, peer-reviewed journals. It will be presented at workshops for patients, healthcare professionals and within local communities.

\section{DISCUSSION}

This study will improve understanding of how to involve patients and/or carers in patient safety in primary care. Extensive stakeholder involvement with patients, carers, GPs, pharmacists and national patient safety organisations has informed the intervention and study codesign. The intervention in this study compromises an introductory workshop to general practice staff and the PSG-PC (both in paper and digital formats). This study is a pragmatic approach to evaluate the PSG-PC in practice. Currently, there is no patient safety tool designed for patients and carers in primary care and as such findings from this study will add value to the evidence base about how to acceptably involve patients and/or carers in patient safety in primary care. ${ }^{141520}$ Given the COVID-19 pandemic, the evaluation will look at the use of the PSG-PC in the context of increased remote consultations.

The findings will need to be evaluated and interpreted considering the following limitations of the study design. The study uses a non-randomised design because of funding limitations and as such the potential influence of general practice settings cannot be controlled for through randomisation. Another limitation is that the PSG-PC has only been developed in English and future work will need to explore how to culturally adapt it. The study will recruit more frequent attenders of general practice; this strategy was chosen pragmatically, however their experiences may be different from less frequent attenders.

Enhancing patient safety in primary care may be supported by involving patients and carers in patient safety for immediate, serious incident prevention to longer term contributions to patient safety include enhancing patient-professional communication to build trust and clarify expectations. ${ }^{7} 50$ This study will determine the feasibility and acceptability of a multicomponent intervention to involve patients and/or carers in patient safety in primary care. This will provide evidence for the acceptability of the PSG-PC to inform future improvements to scale and implement the PSG-PC in primary care.

Correction notice This article has been corrected since it first published. The provenance and peer review statement has been included.

\section{Twitter Rebecca L Morris @beccimorris13}

Acknowledgements We would like to thank all the patient and public contributors, healthcare professionals and researchers who have been involved in advising on the project at different points including: Manoj Mistry, John Jones, Jackie Nightingale, Moira Lyons, Golda Gibson, Carolyn Gamble, Jo Beresford, and all the GPs, pharmacists, members of the public, patients and/or carers who have been 
involved in the virtual involvement groups, the face-to-face meetings and have commented on versions of the patient safety guide.

Contributors RLM and SMC conceived the study. RLM, SMC, CS and KG contributed to the study design. MH provided statistical expertise. RLM, SMC, $\mathrm{CR}$ and $\mathrm{KG}$ developed the intervention. $\mathrm{KG}$ and RLM led the patient and public involvement for the development of the protocol. All authors contributed to the development of the study protocol. RLM led the development of the manuscript, wrote the first draft and led subsequent revisions. RLM, SMC, MH, KG, CR, NS, SJG and CS read the manuscripts and provided critical input. All authors approved the final manuscript.

Funding This study is funded by the National Institute for Health Research (NIHR) Greater Manchester Patient Safety Translational Research Centre.

Disclaimer The views expressed are those of the author(s) and not necessarily those of the NIHR or the Department of Health and Social Care.

Competing interests None declared.

Patient consent for publication Not required.

Provenance and peer review Not commissioned; externally peer reviewed.

Supplemental material This content has been supplied by the author(s). It has not been vetted by BMJ Publishing Group Limited (BMJ) and may not have been peer-reviewed. Any opinions or recommendations discussed are solely those of the author(s) and are not endorsed by BMJ. BMJ disclaims all liability and responsibility arising from any reliance placed on the content. Where the content includes any translated material, BMJ does not warrant the accuracy and reliability of the translations (including but not limited to local regulations, clinical guidelines, terminology, drug names and drug dosages), and is not responsible for any error and/or omissions arising from translation and adaptation or otherwise.

Open access This is an open access article distributed in accordance with the Creative Commons Attribution Non Commercial (CC BY-NC 4.0) license, which permits others to distribute, remix, adapt, build upon this work non-commercially, and license their derivative works on different terms, provided the original work is properly cited, appropriate credit is given, any changes made indicated, and the use is non-commercial. See: http://creativecommons.org/licenses/by-nc/4.0/.

\section{ORCID iDs}

Rebecca L Morris http://orcid.org/0000-0003-1587-0802

Nicola Small http://orcid.org/0000-0002-7879-7967

\section{REFERENCES}

1 Sheikh A, Panesar SS, Larizgoitia I, et al. Safer primary care for all: a global imperative. Lancet Glob Health 2013;1:e182-3.

2 NHS England and NHS Improvement. The NHS patient safety strategy: safer culture, safer systems, safer patients. Available: https://improvement.nhs.uk/documents/5472/190708_Patient Safety_Strategy_for_website_v4.pdf [Accessed Dec 2019].

3 Vincent C, Amalberti R. Safer healthcare: strategies for the real world. Springer, 2016

4 Vosper $\mathrm{H}$, Hignett S, Bowie P. Twelve tips for embedding human factors and ergonomics principles in healthcare education. Med Teach 2018;40:357-63.

5 The Health Foundation. Evidence scan: levels of harm, 2014. Available: http://www.health.org.uk/publications/levels-of-harm/ [Accessed Dec 2019].

6 Aase L, Waring J, Schibevaag. Researching quality in care transitions. Palgrave Macmillan, 2017.

7 Sutton E, Eborall H, Martin G. Patient involvement in patient safety: current experiences, insights from the wider literature, promising opportunities? PMR 2015;17:72-89.

$8 \mathrm{NHS}$ Improvement. Framework for involving patients in patient safety. Available: https://engage.improvement.nhs.uk/policy-strategy-anddelivery-management/framework-for-involving-patients-in-patientsafety/ [Accessed Oct 2020].

9 ledema R, Allen S, Britton K, et al. What do patients and relatives know about problems and failures in care? BMJ Qual Saf 2012;21:198-205.

10 Morris RL, Stocks SJ, Alam R, et al. Identifying primary care patient safety research priorities in the UK: a James Lind alliance priority setting partnership. BMJ Open 2018;8:2

11 Rhodes P, McDonald R, Campbell S, et al. Sensemaking and the co-production of safety: a qualitative study of primary medical care patients. Sociol Health IIIn 2016;38:270-85.
12 Rhodes P, Campbell S, Sanders C. Trust, temporality and systems: how do patients understand patient safety in primary care? A qualitative study. Health Expect 2016;19:253-63.

13 Scott J, Dawson P, Jones D. Do older patients' perceptions of safety highlight barriers that could make their care safer during organisational care transfers? BMJ Qual Saf 2012;21:112-7.

14 Panesar SS, deSiva D, Carson-Stevens A. How safe is primary care? A systematic review. BMJ Qual Saf 2015;0:1-10.

15 De Wet C. An overview of patient safety in primary care. Available: https://www.nes.scot.nhs.uk/media/2075343/an-overview-of-patientsafety-in-primary-care-nov-12.pdf [Accessed Dec 2019].

16 Panagioti M, Khan K, Keers RN, et al. Prevalence, severity, and nature of preventable patient harm across medical care settings: systematic review and meta-analysis. BMJ 2019;366:14185.

17 Daker-White G, Hays R, Esmail A, et al. Maximising involvement in multimorbidity (maximum) in primary care: protocol for an observation and interview study of patients, GPs and other care providers to identify ways of reducing patient safety failures. $B M$ Open 2014;4:8

18 Jerak-Zuiderent S. Certain uncertainties: modes of patient safety in healthcare. Soc Stud Sci 2012;42:732-52.

19 Balogh E, Miller BT, Ball JR. Improving diagnosis in health care. Institute of Medicine. Available: http://www.nationalacademies. org/hmd/Reports/2015/Improving-Diagnosis-in-Healthcare.aspx [Accessed 10 Dec 2019].

20 Allen D, Braithwaite J, Sandall J, et al. Towards a sociology of healthcare safety and quality. Sociol Health IIIn 2016;38:181-97.

21 Heavey E, Waring J, Brun AD. Patients' concepualizations of responsibility for understanding differing attributions in the context of patient safety. J Health Soc Behav 2019:1-16.

22 Davis RE, Jacklin R, Sevdalis N, et al. Patient involvement in patient safety: what factors influence patient participation and engagement? Health Expect 2007;10:259-67.

23 Davis RE, Sevdalis N, Vincent CA. Patient involvement in patient safety: how willing are patients to participate? BMJ Qual Saf 2011;20:108-14.

24 Small N, Bower P, Chew-Graham CA, et al. Patient empowerment in long-term conditions: development and preliminary testing of a new measure. BMC Health Serv Res 2013;13:263.

25 Stafford M, Steventon A, Thorlby R. Briefing: understanding the health care needs of people with multiple health conditions. The Health Foundation, 2018.

26 Kanesarajah J, Waller M, Whitty JA, et al. Multimorbidity and quality of life at mid-life: a systematic review of general population studies. Maturitas 2018;109:53-62.

27 Dixon-Woods M. Why is patient safety so hard? a selective review of ethnographic studies. J Health Serv Res Policy 2010;15:11-16.

28 Elwyn G, Frosch D, Thomson R, et al. Shared decision making: a model for clinical practice. J Gen Intern Med 2012;27:1361-7.

29 Morris RL, Ruddock A, Gallacher K. Developing a patient safety guide for primary care: a co-design approach involving patients, carers and clinicians. Health Expect.

30 NIHR greater Manchester patient safety translational research centre. Available: http://www.patientsafety.manchester.ac.uk/research/ themes/safety-marginalised-groups/ [Accessed Apr 2020]

31 Craig P, Dieppe P, Macintyre S, et al. Developing and evaluating complex interventions: the new medical research council guidance. BMJ 2008;337:a1655.

32 Laugaland K, Aase K, Waring J. Hospital discharge of the elderly-an observational case study of functions, variability and performanceshaping factors. BMC Health Serv Res 2014;14:365.

33 Fetters MD, Curry LA, Creswell JW. Achieving integration in mixed methods designs-principles and practices. Health Serv Res 2013;48:2134-56.

34 Eldridge SM, Lancaster GA, Campbell MJ, et al. Defining feasibility and pilot studies in preparation for randomised controlled trials: development of a conceptual framework. PLoS One 2016;11:e0150205.

35 Chan A-W, Tetzlaff JM, Altman DG, et al. SPIRIT 2013 statement: defining standard protocol items for clinical trials. Ann Intern Med 2013;158:200-7

36 Avery KNL, Williamson PR, Gamble C, et al. Informing efficient randomised controlled trials: exploration of challenges in developing progression criteria for internal pilot studies. BMJ Open 2017;7:e013537.

37 Sluggett JK, Page AT, Chen EYH, et al. Protocol for a nonrandomised pilot and feasibility study evaluating a multicomponent intervention to simplify medication regimens for people receiving community-based home care services. BMJ Open 2019;9:e025345.

38 Patton MQ. Qualitative evaluation and research methods. Sage Publications, 2002 
39 Hernan AL, Giles SJ, O'Hara JK, et al. Developing a primary care patient measure of safety (PC PMOS): a modified Delphi process and face validity testing. BMJ Qual Saf 2016;25:273-80.

40 Beecham J, Knapp M. Costing psychiatric interventions. In: Thornicroft G, ed. Measuring mental health needs, Gaskell. 2nd edn, 2001: 200-24.

41 Herdman M, Gudex C, Lloyd A, et al. Development and preliminary testing of the new five-level version of EQ-5D (EQ-5D-5L). Qual Life Res 2011;20:1727-36.

42 Green J, Thorogood N. Qualitative methods for health research. London: Sage, 2004.

43 Mays N, Pope C. Assessing quality in qualitative research. BMJ 2000;320.

44 Murray E, Treweek S, Pope C, et al. Normalisation process theory: a framework for developing, evaluating and implementing complex interventions. BMC Med 2010;8:63.
45 Mair FS, May C, O'Donnell C, et al. Factors that promote or inhibit the implementation of e-health systems: an explanatory systematic review. Bull World Health Organ 2012;90:357-64.

46 Creswell JW, Plano Clark VL. Designing and conducting mixed methods research. Sage Publications, 2017.

47 Ivankova NV, Creswell JW, Stick SL. Using mixed-methods sequential explanatory design: from theory to practice. Field Methods 2006;18:3-20.

48 INVOVLE. Payment and recognition of public involvement. Available: https://www.invo.org.uk/resource-centre/payment-and-recognitionfor-public-involvement/ [Accessed Dec 2019].

49 Health Research Authority. Guidance about Covid-19 for sponsors, sites and researchers. Available: www.hra.nhs.uk/coronavirus [Accessed Apr 2020].

50 Stocks SJ, Donnelly A, Esmail A. Frequency and nature of potentially harmful preventable problems in primary care form the patient's perspective with clinician review: a population-level survey in Great Britain. BMJ Open 2018;8. 